\title{
Anatomy and Histology of the Human and Murine Prostate
}

\author{
Michael Ittmann \\ Department of Pathology and Immunology, Baylor College of Medicine, Houston, Texas 77030 \\ Correspondence: mittmann@bcm.edu
}

The human and murine prostate glands have similar functional roles in the generation of seminal fluid to assist in reproduction. There are significant differences in the anatomy and histology of murine and human prostate and knowledge of the normal anatomy and histology of the murine prostate is essential to interpreting changes in genetically engineered mouse models. In this review, the normal anatomy and histology of both human and mouse prostate will be described.

\begin{abstract}
Analysis of molecular alterations in human Aprostate cancer tissues has been critical in understanding the molecular basis of prostate cancer. Initially, changes were analyzed for single genes, proteins, or genomic regions. However, over the last decade, high-throughput methodologies have allowed examination of genetic and epigenetic changes and associated changes in gene expression in prostate cancer on an unprecedented scale for both clinically localized and metastatic disease. Although there are some disease states that not well characterized to date (i.e., metastatic treatment naïve cancer), overall, these efforts have allowed for the beginning of a clinically and biologically meaningful classification of prostate cancer.

Ultimately, analysis of molecular alterations in human cancer tissues is descriptive, and functional studies are critical in defining the underlying biology induced by the genetic and epigenetic changes seen in the cancer tissues. Mouse models have played a central role in defining the
\end{abstract}

biological importance of the annotated lesions in human prostate cancer by engineering the described lesions into mouse models (Ittmann et al. 2013). Genetically engineered models have significant advantages in that they reflect tumor progression over time from the initiation of preinvasive lesions to invasive and, in some cases, metastatic lesions within the prostatic microenvironment, including a fully intact immune system. Particularly with the increasing importance of immunotherapy, the latter is a key advantage over xenograft models.

\section{ANATOMY AND HISTOLOGY OF THE HUMAN PROSTATE}

The human prostate gland is a pyramid-shaped organ located beneath the bladder with the apex (corresponding to apex of the pyramid) contacting the penile urethra and the base contacting to the bladder (Paner 2010). The prostate lies below the urinary bladder and is located in front of the

Editors: Michael M. Shen and Mark A. Rubin

Additional Perspectives on Prostate Cancer available at www.perspectivesinmedicine.org

Copyright (C 2018 Cold Spring Harbor Laboratory Press; all rights reserved; doi: 10.1101/cshperspect.a030346

Cite this article as Cold Spring Harb Perspect Med 2018;8:a030346 
M. Ittmann

rectum. The prostate surrounds the prostatic urethra that is the conduit for urine flow from the bladder. The normal prostate weighs about 15-20 g. The seminal vesicles are located bilaterally at the base of the prostate. The human prostate is a single gland with different histological zones (peripheral, transition, and central zones). As shown in Figure 1, the peripheral zone is wrapped around the outer portion of the prostate distally and is the site of origin of the majority of prostate cancers. It constitutes about $70 \%$ of tissue in the normal prostate (Paner 2010). The transition zone is located near the prostatic urethra and is inconspicuous in most young men, constituting about $5 \%$ of the prostate. In the majority of older men, the transition zone is enlarged considerably by benign prostatic hyperplasia, an extremely common benign proliferation in transition zone tissue. Most radical prostatectomy specimens show evidence of variable degrees of benign prostatic hyperplasia (see Fig. 2, for example). Cancers also arise in the transition zone and there is considerable evidence that cancers arising in the transition zone are clinically and biologically different than peripheral zone cancers, although there is considerable overlap. The central zone is a cone-shaped region, with the wider portion at the base of the prostate and the apex at the verumontanum surrounding the ejaculatory ducts. It is not the site of origin of any disease process but of course can be secondarily involved by cancer.

The human prostate glandular epithelium is composed of acini and ducts lined by three types of cells: luminal, basal, and neuroendocrine (see Fig. 1C). The acini have an undulating to papillary appearance in most cases (Fig. 2A-D). This papillary configuration is noticeably more pronounced in the central zone (Paner 2010). The luminal cells are columnar, with pale eosinophilic cytoplasm and round nuclei near the base of the cell (Fig. 2D). Luminal cells are specialized cells that secrete a variety of products into the lumen, which contribute to the formation of the seminal fluid. These products include prostate-specific antigen (PSA), and luminal cells are strongly positive for PSA immunohistochemistry. Basal cells are adjacent to the basement membrane and have ovoid nuclei and inconspicuous cytoplasm. The number of basal cells can be variable between glands in an individual prostate. They can generally be identified by careful examination of routine $\mathrm{H} \& \mathrm{E}$ sections but are easier to identify using immunohistochemistry for p63 (nuclear) and high-molecular cytokeratins (cytoplasmic). Neuroendocrine
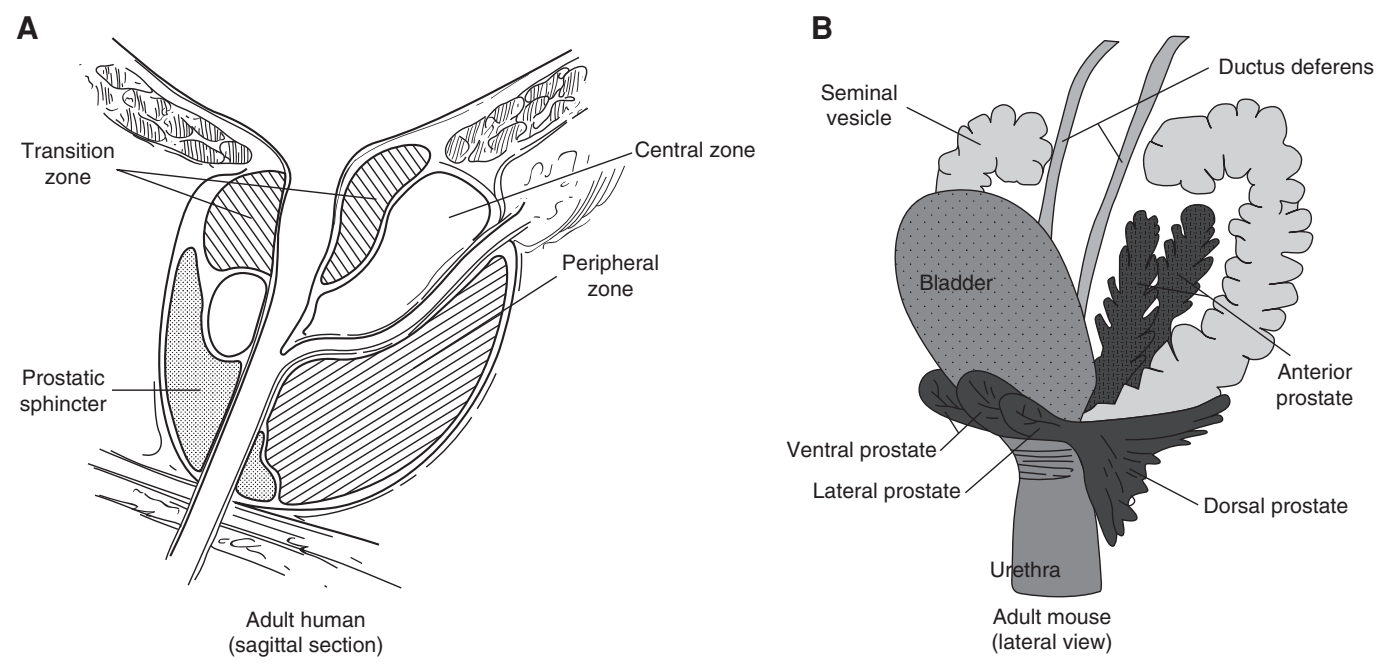

Figure 1. Schematic illustration of human and mouse prostate and adjacent structures. $(A)$ Human prostate showing the location of the three zones of the prostate. (B) Mouse prostate showing different lobes of the prostate and their relation to adjacent structures. 

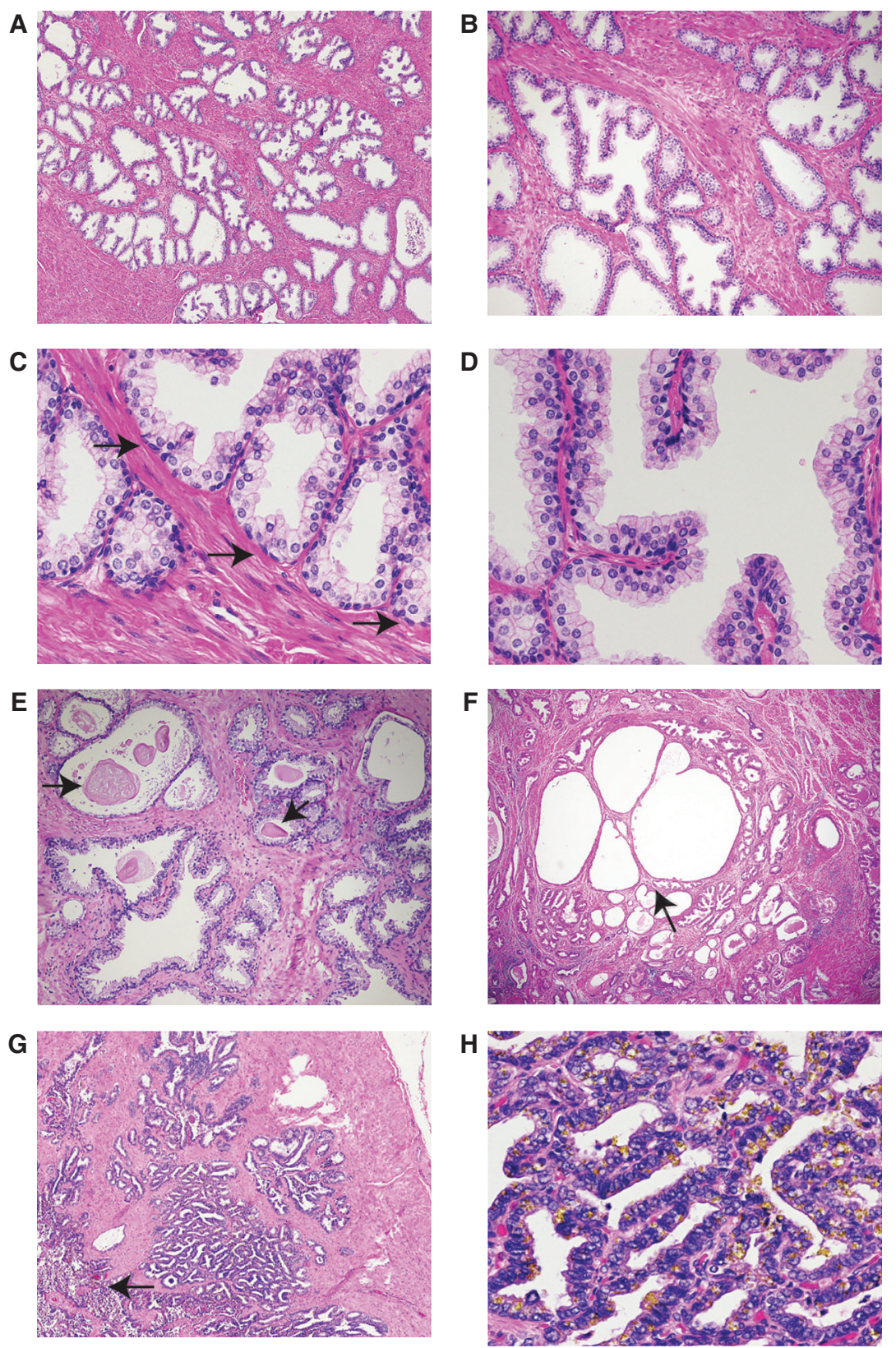

Figure 2. Human prostate and seminal vesicles. $(A)$ Low, $(B)$ medium, and $(C, D)$ high-power views of the peripheral zone of the human prostate. Note the loosely lobulated arrangement of acini $(A, B)$. Basal cells are characterized by ovoid nuclei and lie near the basement membrane ( $C$, arrows). The acini can have small papillae $(D)$. (E) Laminated corpora amylacea are commonly seen with the prostatic acini (arrows). (F) Benign prostatic hyperplasia is characterized by nodular proliferation of epithelium and stroma. This nodule shown at low power has both epithelial and stromal hyperplasia. Cystic atrophy is common in hyperplastic nodules (arrow). Chronic inflammation is present in the lower left. $(G)$ Low power of seminal vesicle with central lumen indicated by an arrow with numerous smaller glandular structures. $(H)$ High-power view of seminal vesicle; the abundant yellow liposfuschin pigment is epithelium. The amount of pigment is variable and in this example is particularly abundant. 
M. Ittmann

cells cannot be reliably identified on H\&E sections but can be highlighted by immunohistochemistry for neuroendocrine markers such as chromogranin and synaptophysin. Rounded laminated eosinophilic corpora amylacea are commonly seen within the lumens of the acini (Fig. 2E).

The prostatic stroma is fibromuscular, with abundant smooth muscle cells admixed with fibroblasts, blood vessels, and nerves. No adipose tissue is present in the prostate. This fibromuscular stroma is much more prominent than the relatively thin fibromuscular stroma in the mouse prostate. Skeletal muscle fibers are predominantly outside the prostate but often extend into the outer portion of the prostate as well.

As noted above, most prostates removed for the treatment of prostate cancer show variable degrees of benign prostatic hyperplasia such that the majority of tissue in most radical prostatectomies shows benign prostatic hyperplasia. This is manifested by nodular proliferation of tissue with variable components of epithelium and stroma, ranging from predominantly epithelial to pure stromal nodules (Fig. 2F).

The human prostate commonly shows a wide variety of alterations in the epithelium and stroma. Atrophy of the epithelium is extremely common as is basal cell hyperplasia. Chronic inflammation is also extremely common and variable degrees of acute inflammation from focal to extensive with abscess formation can be seen. Infarcts, in which tissues die owing to compromise of the blood supply, can be seen in areas of benign prostatic hyperplasia, and squamous metaplasia can be present at the edges of these infarcts.

The seminal vesicles in humans are bilateral tubular structures with a central lumen into which empties a complex of smaller glands, all lined by a columnar epithelium with eosinophilic cytoplasm (Fig. 2G). Characteristic yellow lipofuschin pigment is present in the epithelial cells (Fig. 2H). The nuclei can be hyperchromatic and display degenerative atypia. The epithelium is surrounded by prominent smooth muscle layers with an inner circular and outer longitudinal layer. Primary neoplasia of the seminal vesicles is exceedingly rare (Paner
2010) but invasion of one or both seminal vesicles by prostatic adenocarcinoma is not uncommon and is associated with a significantly worse prognosis.

\section{ANATOMY AND HISTOLOGY OF MOUSE PROSTATE}

The mouse prostate consists of four lobes: the anterior, ventral, dorsal, and lateral lobes (Fig. 1B). The latter two are sometimes grouped together as the dorsolateral lobe. The anterior lobe is also known as the coagulating gland. It is bilateral and located cranial to the other lobes. The anterior lobes are elongated and tubular and attached to the lesser curvature of the seminal vesicles. Grossly, they are thin and glistening. The ventral lobe is a single structure located ventrally in the midline above the urethra. It is also thin and delicate. The dorsal lobe is located dorsally near the urethra and is flanked on each side by the lateral lobes (Knoblaugh and True 2012).

The lining epithelium of all lobes consists of secretory epithelial cells, basal cells with minimal cytoplasm located beneath the secretory epithelial cells, and a small number of neuroendocrine cells as in the human prostate. The latter cells are not easily identifiable by routine histology.

The lobes are covered by a thin mesothelium that also contains adipose tissue, nerves, and blood vessels. The glands of each lobe have a distinct histology. They are also surrounded by stroma of different thicknesses in different lobes. Based on immunohistochemistry with CD-34 and smooth muscle actin (SMA) and location, four stromal subtypes have been identified (Peng et al. 2013). In addition to smooth muscle cells $\left(\mathrm{CD} 34^{+} / \mathrm{SMA}^{+}\right)$, there are three distinct populations of $\mathrm{CD} 34 / \mathrm{SMA}^{-}$fibroblastic or fibroblastlike cells. These three types are: subepithelial cells, wrapping cells (tightly associated with the outer surface of smooth muscle cells), and interstitial fibroblasts (between ducts). The first two cell types along with the smooth muscle cells constitute the duct wall. The histology of each lobe is shown in Figure 3. The ventral prostate has moderate-to-large acini lined by cubodal cells with sparse infolding and tufting (Fig. $3 \mathrm{~A})$. The stroma is very thin. The secretions are 


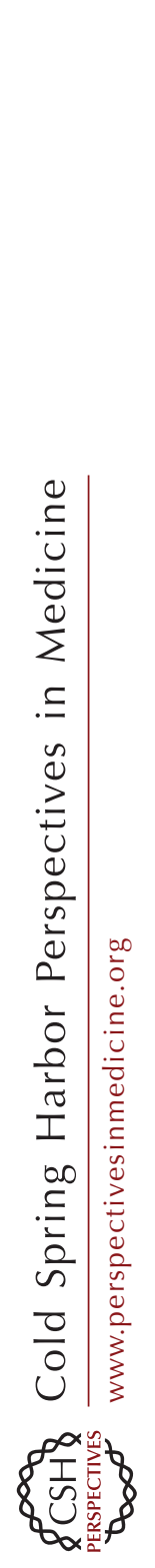

A

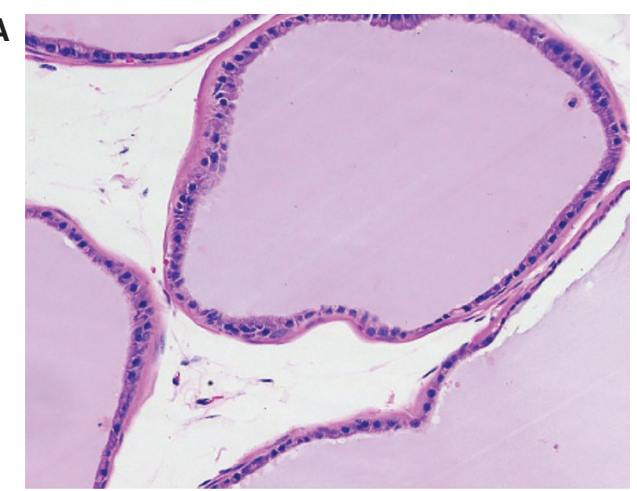

C

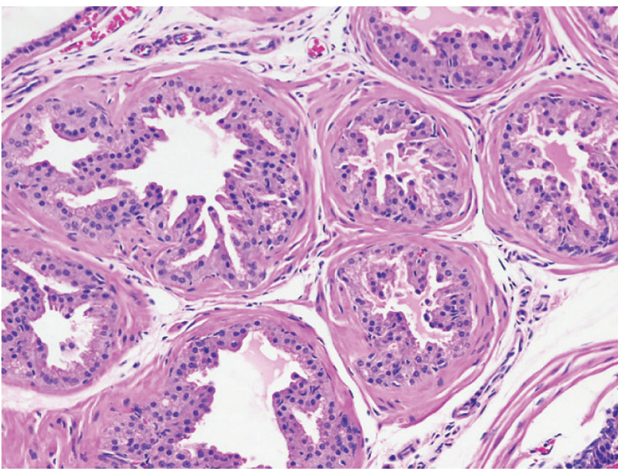

E

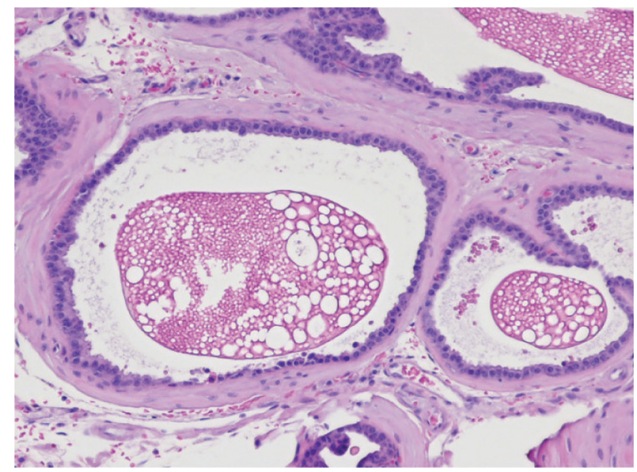

B

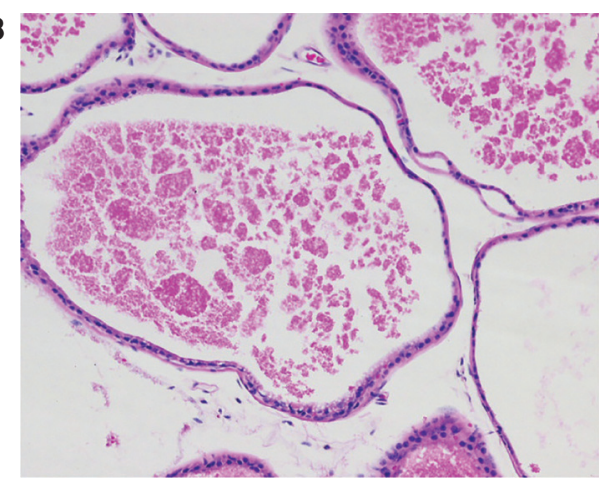

D

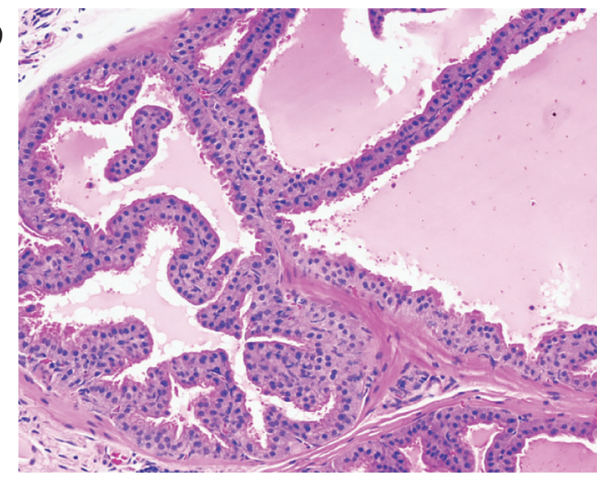

$\mathbf{F}$

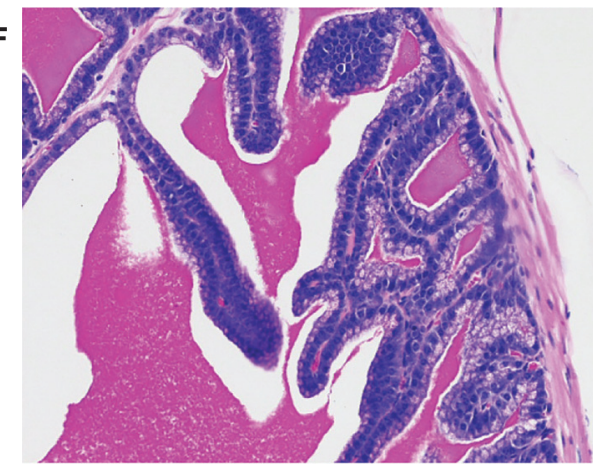

Figure 3. Mouse prostate and adjacent structures. $(A)$ Ventral prostate. Note flat epithelium and bluish tinged secretion. Stroma is thin. $(B)$ Lateral prostate. Flat epithelium with this stroma with characteristic clumped eosinophilic secretions. $(C)$ Dorsal prostate. Papillated epithelium with thicker fibromuscular stroma. $(D)$ Anterior prostate. Papillated epithelium with more intensely eosinophilc cytoplasm. (E) Ampullary gland. Flat epithelium with characteristic "Swiss cheese" appearance of secretions. (F) Seminal vesicle. Papillated epithelium with intensely eosinophilic secretions. All at 200× magnification.

distinctive in that they are homogeneous and have a slight blue tinge that is not seen in the other lobes. The nuclei are basal. The lateral prostate has generally larger acini lined by cuboidal cells with sparse infolding (Fig. 3B). The fibromuscular stroma is thin. The secretions are eosinophilic and often clumpy. Nuclei are basally located. The dorsal prostate consists of relatively small acini lined by columnar epithelial cells with infolding and eosinophilic cytoplasm (Fig. 3C). The dorsal prostate has a noticeably thicker stroma than the other lobes and the se- 
M. Ittmann

cretions are homogeneous and lightly eosinophilic. The nuclei are centrally located. The anterior prostate is lined by a cuboidal to columnar epithelium with a more eosinophilic cytoplasm and a papillary growth pattern (Fig. 3D). The cytoplasm has a slight granularity and nuclei are centrally located. Based on the above, each lobe can be recognized based on its distinctive histology, although when the lobes are involved with pathological processes this can be problematic.

Other structures adjacent to the prostate are of some importance because they can be secondarily involved in pathological processes arising in the prostate and/or be the site of primary pathology in genetically engineered models. The ampullary glands are bilateral outpouchings from the proximal ductus deferens and are of mesodermal origin. It does not have a human counterpart (Knoblaugh and True 2012). The acini are small and are mainly flat with slight infolding (Fig. 3E). The lining cells are cuboidal, eosinophilic, and have basal nuclei. The stroma is similar in thickness to the dorsal prostate. The most characteristic feature of the ampullary gland is the dense secretions with a "Swiss cheese" or bubbly appearance.

The two seminal vesicles are dorsolateral to the bladder. The seminal vesicles are lined tall columnar cells with large intricate branching folds and are surrounded by a modest fibromuscular stroma (Fig. 3F). The epithelial cells have basal nuclei that are surrounded by hematoxyphilic cytoplasm with apical pale vacuolated cytoplasm that is distinctive. The secretions are homogeneous, dense, and brightly eosinophilic. The amount of secretion is variable, and when the seminal vesicle is distended the folds are flattened considerably.

Other structures frequently seen near the prostate include the ductus deferens, ganglia, and their associated nerves, and of course the bladder and the membranous urethra.

\section{CONCLUDING REMARKS}

As described above the human and murine prostate have considerable differences in anatomy and histology despite a similar functional role in reproduction. To date, there is no firm evidence that any specific murine prostate lobe is more valid as a model for human prostate cancer and models have been described involving every lobe and, frequently, multiple lobes. Although anatomically and histologically different than human prostate, there is extensive evidence that the genetic lesions in human prostate cancer can lead to neoplasia or neoplastic progression in murine prostate, either alone or in combination when engineered in mouse prostate. The differences in anatomy and histology between human and mouse prostate and the associated structures must always be taken into account in the pathological analysis of genetically engineered mouse models.

\section{REFERENCES}

Ittmann M, Huang J, Radaelli E, Martin P, Signoretti S, Sullivan R, Simons BW, Ward JM, Robinson BD, Chu GC, et al. 2013. Animal models of human prostate cancer: The consensus report of the New York meeting of the Mouse Models of Human Cancers Consortium Prostate Pathology Committee. Cancer Res 73: 2718-2736.

Knoblaugh S, True L. 2012. Male reproductive system. In Comparative anatomy histology. A mouse and human atlas (ed. Treuting P, et al.), pp. 295-303. Elsevier, Amsterdam.

Paner G. 2010. Prostate gland and seminal vesicle. In Diagnostic pathology: Genitourinary (ed. Amin M, et al.), Section 3, pp. 4-156. Amirysis, Salt Lake City, UT.

Peng YC, Levine CM, Zahid S, Wilson EL, Joyner AL. 2013. Sonic hedgehog signals to multiple prostate stromal stem cells that replenish distinct stromal subtypes during regeneration. Proc Natl Acad Sci 110: 20611-20116. 


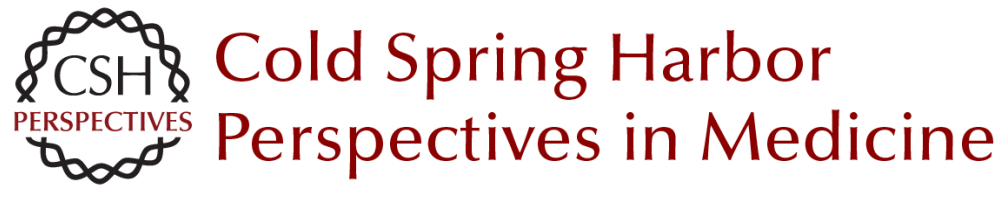

\section{Anatomy and Histology of the Human and Murine Prostate}

Michael Ittmann

Cold Spring Harb Perspect Med 2018; doi: 10.1101/cshperspect.a030346 originally published online October 16, 2017

\section{Subject Collection Prostate Cancer}

Anatomic and Molecular Imaging in Prostate Cancer

Eric T. Miller, Amirali Salmasi and Robert E. Reiter

The Epidemiology of Prostate Cancer Claire H. Pernar, Ericka M. Ebot, Kathryn M. Wilson, et al.

Prostate Stem Cells and Cancer Stem Cells Jia J. Li and Michael M. Shen

Prostate Cancer Epigenetics: From Basic Mechanisms to Clinical Implications Srinivasan Yegnasubramanian, Angelo M. De Marzo and William G. Nelson

\section{The Genomics of Prostate Cancer: A Historic} Perspective Mark A. Rubin and Francesca Demichelis

Neuroendocrine Differentiation in Prostate

Cancer: Emerging Biology, Models, and Therapies Loredana Puca, Panagiotis J. Vlachostergios and Himisha Beltran

DNA Damage Response in Prostate Cancer Matthew J. Schiewer and Karen E. Knudsen

Transcriptional Regulation in Prostate Cancer David P. Labbé and Myles Brown
New Opportunities for Targeting the Androgen Receptor in Prostate Cancer Margaret M. Centenera, Luke A. Selth, Esmaeil Ebrahimie, et al.

Prostate Cancer Research at the Crossroads Michael M. Shen and Mark A. Rubin

Immunotherapy for Prostate Cancer Nicholas J. Venturini and Charles G. Drake

Molecular Pathology of High-Grade Prostatic Intraepithelial Neoplasia: Challenges and Opportunities Levent Trabzonlu, Ibrahim Kulac, Qizhi Zheng, et al.

Metastases in Prostate Cancer Federico La Manna, Sofia Karkampouna, Eugenio Zoni, et al.

Genetically Engineered Mouse Models of Prostate Cancer in the Postgenomic Era Juan M. Arriaga and Cory Abate-Shen

Molecular Biomarkers in the Clinical Management of Prostate Cancer Aaron M. Udager and Scott A. Tomlins

Metabolic Vulnerabilities of Prostate Cancer: Diagnostic and Therapeutic Opportunities Giorgia Zadra and Massimo Loda

For additional articles in this collection, see http://perspectivesinmedicine.cshlp.org/cgi/collection/ 